\title{
Is quantum theory falsified by loophole-free Bell experiments?
}

\author{
Ghenadie N. Mardari ${ }^{1}$ **
}

1 Open Worlds Research, Sparks, Maryland, USA

* Correspondence: gmardari@gmail.com

\begin{abstract}
Quantum theory predicts a whole class of non-local phenomena, observable via coincident detection of EPR-type systems. An important feature of these observations is their non-signaling character. Technically, non-local phenomena should only be observable for post-selected sub-ensembles, rather than for complete projections. Otherwise, superluminal telegraphy becomes possible. Yet, a couple of recent Bell experiments reported the observation of quantum non-locality for $100 \%$ of the detected events. Does it follow that signaling non-locality is possible? If so, was quantum theory falsified? This puzzle is solved by revisiting the interpretation of the spin projection operator, with special focus on its dual nature (combining spectral decomposition with spectral transformation). "Component switching" is not a loophole, but rather a requirement of quantum mechanics in this context, because sharp spin projections are partial (as well as partially overlapping). Surprisingly, it is possible to pre-select incompatible statistical sub-ensembles with heralded detection and to reveal the same behavior as in post-selected observations. Therefore, Bell experiments confirm the predictions of quantum theory without violating the non-signaling principle.
\end{abstract}

Keywords: Bell's theorem; EPR paradox; quantum entanglement; non-locality.

\section{Introduction}

Quantum Bell experiments have been at the cutting edge of scientific innovation for the past five decades [1, 2]. Given the risk of interpretive ambiguity, they need to be designed in highly constrained ways, with no room for reasonable "loopholes" [3, 4]. As a rule, these tests are expected to either confirm the validity of quantum theory by violating a Bell-type inequality, or to undermine its foundations with a negative result. However, a third outcome is also possible. Namely, an experiment can both violate a Bell-type inequality and falsify quantum theory. After all, some aspects of quantum theory might seem "strange", but not everything that is "strange" is part of quantum theory. For example, superluminal telegraphy is a topic of high interest in the science fiction community, but it has no support in quantum theory. Yet, it is precisely this possibility that was apparently confirmed by two recent loophole-free Bell experiments [5, 6]. Thus, it is necessary to understand: was quantum theory falsified, or - if not - what else can explain these outcomes?

Quantum non-locality is a complicated concept, and it is still a topic of heated debates. To this day, there is no consensus as to what exactly it is. However, it is quite straightforward to specify what it is not, at least in one regard. Namely, it is not a platform for superluminal messaging. Quantum non-locality is non-signaling [7-9]. Though, what is the physical meaning of this? Does quantum non-locality require physical interactions between entangled quanta, or is it just a consequence of their strong correlation? The answer depends on whether quantum theory predicts Bell violations for identical sub-ensembles, or whether it requires "component switching" between alternative measurements. If we assume that the first alternative is correct, then we get interpretive paradoxes and the unexpected implication that quantum non-locality is signaling. Unfortunately, this assumption is widely perceived as correct, and even inspired the design of numerous "loophole-free" experiments [5-6, 10-13]. The goal of this essay is to explore the subtleties this topic, and to identify the interpretive misconceptions that lead to this dramatic distortion of quantum theory. 
When it comes to Bell experiments, the main source of confusion can be traced to the unusual features of spin $1 / 2$ quantum variables. On the one hand, spin vectors are superposed with each other. If so, complex profiles should allow for decomposition into welldefined components, just like other quantum properties (such as, frequency spectra) [1416]. Accordingly, every sharp measurement should target a different ensemble of quanta from a common input projection. On the other hand, spin vectors do not commute with each other $[17,18]$. If so, different observables should correspond to different profiles of a single wave-function (as seen, for example, in the case of momentum and position spectra). In this case, every measurement should target one and the same group of quanta. Yet, how is it possible for both of these conclusions to be true? It makes no sense for a measurement to target a single component and the full projection at the same time. As a matter of established practice, this apparent contradiction is removed by re-interpreting quantum superposition. Instead of describing a system as being in all the component vectors at the same time, it is described as if it is fluctuating randomly between them. For example, in the case of photon polarization, the usual assumption is that alternative quantum measurements divide the full population 50-50 between two orthogonal vectors. The implication is that spin $1 / 2$ measurements sample complete input projections, which is a gross distortion of the quantum formalism, and is in flat contradiction with the experimental evidence. Not surprisingly, this interpretation produced the expectation of Bell violations for identical sub-ensembles, allowing for signaling non-locality. In contrast, it is more plausible to suggest that spin projections are both partial and partially over-lapping, because quantum non-commutativity is variable for this type of observables [19]. As will be shown below, this step eliminates the paradoxes that otherwise emerge both in theoretical and in experimental studies.

In quantum experiments with unrestricted measurement schemes, non-local phenomena become possible when small sub-ensembles of coincident events are post-selected from larger ensembles of single events. This statement was apparently falsified by two recent experiments [5, 6], because they achieved Bell violations for $100 \%$ of detected events, apparently without shortcuts. Though, does it follow that quantum theory is wrong and that signaling non-locality is possible? As will be shown below, the answer is negative, because these experiments adopted a restricted measurement scheme. They used heralded detection to perform partial scanning of temporal distributions. This enabled them to manipulate the range of detectable events for each joint measurement. In essence, this is a demonstration that "loophole-free" experiments can have loopholes. Yet, these are not loopholes around quantum theory. Instead, they are loopholes required by quantum theory to produce the false appearance of signaling non-locality. The build-up to this conclusion is presented below. Section 2 reviews the background for the established interest in "fair sampling" for Bell experiments, explaining the connection between the socalled "detection loophole" and the drive to avoid post-selection in loophole-free Bell experiments. Section 3 explores the nuances of quantum superposition, with emphasis on the link between the virtual components of an input projection and the statistical ensembles at the output of sharp measurements. The main bottleneck for the study of quantum entanglement is discussed in Sections 4-6. In the case of spin $1 / 2$ variables, there is a major gap between facts and opinions in the physics community. The theory describes partial measurements; the experiments are carefully designed to isolate relevant components; yet both the critics and the supporters of non-locality believe that these measurements are complete. Hence, the "strangeness" of quantum theory is a result of its distortion in practice, rather than a fundamental ontological problem. With this in mind, Section 7 addresses the appearance of signaling non-locality in loophole-free experiments and explains their underlying mechanism. Finally, section 8 is a discussion of the concept of nonlocality in its theoretical context. In retrospect, it seems to have worked as a distraction, diverting research away from the challenge to reconcile the basic principles of quantum theory, especially as they relate to various aspects of quantum superposition. 


\section{Fair sampling and Bell experimentation}

A typical Bell experiment is performed by conducting four alternative joint measurements $[15-18,20]$. The goal is to obtain four coefficients of correlation and to determine if they can belong to a single population or not. When multiple coefficients of correlation are compatible with each other, they obey a corresponding Bell inequality (such, as the CHSH inequality [21]). When they are incompatible (and therefore belong to different populations), they can violate such inequalities. In the context of quantum entanglement, Bell experiments are meant to serve as tests of locality. If one and the same population, measured in four different ways, produces incompatible pairwise correlations, then it must be true that quanta change properties from measurement to measurement. Yet, when a single quantum (say, in Geneva) changes its properties because another quantum (say, in Vienna) was measured differently, then some kind of non-local influence is required to explain the observation. Accordingly, Bell tests are designed and carried out with the intention to verify the reality of non-local interactions at the quantum level.

The first indisputable confirmation of Bell violations in quantum mechanics was produced by Aspect and his collaborators, in a series of experiments in the early 1980's [2224]. Those tests revealed conclusively that Bell inequalities are violated by entangled quanta. Unfortunately, they couldn't offer sufficient proof that non-local interactions were real. The problem was that joint measurements were post-selected at a very low rate [25]. Roughly speaking, only one in a million quanta at each detector had a coincidentally detected partner at the other detector. Therefore, it was very hard to argue that one and the same one-millionth of the output projection was post-selected in each of the four joint measurements. (This would be like winning the lottery four times in a row with the same number). If the sampled (sub)populations are not necessarily identical, then the observed behavior is not necessarily non-local. To account for this possibility, Aspect and collaborators insisted that non-locality is still verified, under the assumption that quantum theory is non-local and the obvious agreement between theory and experiment in their demonstrations. Yet, they had to add an additional assumption, known as "fair sampling", suggesting that different iterations were indeed targeting the same population, even though the rate of post-selection was so low.

This discrepancy between detected and post-selected quanta became known as the "detection loophole" and attracted a lot of attention. As the name implies, the problem was blamed on experimental shortcomings, and the priority was to solve it with technological improvements. Over time, progress was made with the advent of detectors with higher efficiency, improved coatings for optical devices, and by replacing free-space setups with fiber optic channels and solid-state devices $[18,20]$. The benefit of these developments is undeniable. However, insofar as the goal is to unveil the nature of quantum reality, this project was a red herring, because the specified effect was too large to be accounted for by experimental losses. For instance, if we assume that $99 \%$ of all the photons were lost in transit between source and detector, and if we also assume that detector efficiency is $1 \%$, we still expect a maximal mismatch of only $1 \times 10000$ between single and coincident events. Even with these ridiculous assumptions, the signal would have to be 100 times smaller than the background noise to explain the observed rates of coincidence, which would make it impossible to observe any quantum effect with a reasonable measure of consistency. More importantly, the rates of coincident detection would have been dramatically higher, if all the entangled quanta were indeed targeted for detection. After all, Bell experiments are designed to test for correlations between fixed points from wide projections. Most of the detected single quanta in the "Alice" path have matching partners in the "Bob" path, but these partners land on other points (not the single point selected for analysis). In other words, the low rates of coincidence are obtained by design. They are expected even in ideal lossless experiments, as will be shown in greater detail below, because they are required by quantum theory.

The necessarily partial nature of Bell measurements is conspicuously absent in public debates about quantum non-locality. Nonetheless, this problem was addressed in narrow expert circles. What we know today from private conversations at conferences is that the 
possibility of "component switching" was perceived as incompatible with quantum theory, where "no sub-ensembles are possible". Furthermore, as shown by Larsson in 1998, any concern about fair sampling could be removed if the ratio of coincident to single events was raised enough to reach a predictable threshold [26]. Namely, if this ratio was $82.8 \%$ or higher, it did not matter if the losses were at the detector, in transit, or even by design. Significant violations of the CHSH inequality would automatically rule out local explanations, because the four coefficients of correlation would necessarily belong to a common population.

For the sake of clarity, consider an experimental context, in which Alice and Bob detect entangled quanta, but their rate of coincidence is $50 \%$. If Alice detects four quanta, then only two of them produce coincidences. If Bob makes measurement $\boldsymbol{b}$, quanta $\# 1$ and $\# 3$ might be post-selected. If Bob makes measurement $\boldsymbol{b}^{\prime}$, quanta \#2 and \#4 might be in play instead. Hence, it is possible to obtain incompatible coefficients of correlation without non-locality if every joint measurement isolates a different sub-population. In contrast, if the rate of coincidence is $100 \%$, then it is always the same set of quanta that generates observables (i.e., all of them). In this case, incompatible correlations would indicate a change in the physical profile for non-local reasons. Moreover, an intermediate scenario is also possible, where the rate of coincidence is not $100 \%$, and still - given the strength of achievable Bell violations - the outcome is reducible to a single population. Consequently, it should be possible to prove the reality of "fair sampling" with experimental settings that are achievable in practice.

For several generations, Bell experiments were regularly repeated, every time a new technological breakthrough enabled detection improvements. This meant that progress was incremental and slow. Yet, the pinnacle of this long project was a flurry of loopholefree Bell experiments in the recent hears [5, 6, 10-13]. More importantly, two of these experiments achieved the ideal level of $100 \%$ coincidence with the CHSH protocol, while also closing other loopholes as well. In one experiment, this was achieved by entangling nitrogen vacancies in diamond crystals [5]. In another, rubidium atoms were used [6]. Nonetheless, the CHSH inequality was violated significantly. Thus, any possible experimental shortcoming became irrelevant. Once the rate of coincidence is perfect, fair sampling must be assumed. Therefore, all the theories that might predict Bell violations for non-identical sub-ensembles are falsified. Ergo, quantum behavior should be interpreted as non-local. There is just one problem: this sort of behavior automatically allows for signaling non-locality, contrary to the known features of quantum theory. Consequently, these experiments appeared to falsify classical mechanics and quantum mechanics in equal measure. Instead, they appeared to support a separate class of theories that support superluminal signaling. After all, if every quantum is part of a coincidence, then it is not necessary to post-select anything. Raw data can exhibit these phenomena directly, at least for some types of quantum phenomena. For example, "ghost imaging" [27, 28] would allow for superluminal image and video broadcasting, while "quantum erasure" [29] would enable communication into the past, or even into the future. Bell experiments are more subtle in this regard because they test for indirect effects (coefficients of correlation). However, these settings would also allow for superluminal messaging when scaled up to systems with 3 or more entangled quanta [30]. So, how can it be that an experiment designed to verify the predictions of quantum theory ended up with a result that both confirms and falsifies it? The best assumption is that something must be missing in this description. The goal of this essay is to explore this problem and to propose a simple solution.

\section{Physical and statistical sub-ensembles in quantum mechanics}

Quantum superposition may sound counterintuitive, from a classical point of view, but it is nonetheless conceptually straightforward. This can be seen already in the textbook example of the double-slit experiment. Classical intuitions might suggest the reality of two well-defined projections, one from each slit. These projections should interact with each other and produce interference fringes. Yet, quantum experiments show 
unambiguously that single quanta, propagating one at a time, can produce the same patterns, once enough of them are recorded on a screen. Ergo, quanta cannot belong to meaningful sub-ensembles that correspond to individual paths for propagation (Fig. 1a). Instead, the context of observation determines the parameters of the relevant wave-function, and only the net state of this wave-like process can explain the observable properties of single quanta. In short, single quanta are always in the net state of the whole system to which they belong, and they can only have observable properties that reflect such states at any chosen coordinate for observation.

The difference between quantum superposition and classical superposition has remarkable statistical implications. For example, in the classical systems, superposition is described as a collective effect in which every microscopic component is represented by a well-defined "sub-ensemble" of detectable objects. In the quantum case, every single object is expected to be in the net state of the whole system. In other words, there are no physical sub-ensembles in quantum mechanics in this sort of contexts. Accordingly, to study the relationships between the components of a classical superposed system, it is necessary to isolate and to sample the corresponding physical sub-ensembles. Therefore, classical measurements of this sort are presumed to be statistically and physically partial. In contrast, it is impossible to isolate any physical sub-ensemble for the study of quantum systems. Instead, it is necessary to sample the net state of the full quantum projections. Thus, sharp quantum measurements are presumed to provide information about complete statistical profiles. For visual illustration, consider a mixed classical beam with numerous polarization components (vertical, horizontal, and every value in-between). A sharp measurement of polarization would isolate a narrow slice of the input beam, effectively filtering out all the photons that are not in the selected state. In contrast, a quantum beam contains only quanta in the net state of the projection. It does not matter which value is chosen for observation, since every quantum is a potential candidate for observation. Thus, it seems reasonable to presume that every sharp spin $1 / 2$ measurement should divide the input projection 50-50, in full. The implicit assumption of Bell's Theorem is that sharp quantum measurements express full population statistics. Consequently, they should be compared with classical joint distributions that are possible in complete populations, and not with disjoint distributions that apply to classical sub-ensembles. Presumably, if there are no physical sub-ensembles in quantum projections, there can be no statistical sub-ensembles. Yet, this is not the full picture.

Consider an optical beam, created by mixing three types of frequencies: reg, green and blue (Fig. 1b). The three components are indistinguishable, and therefore we have a beam that is observable as "white" light. According to the rules that govern quantum superposition, no single quantum can be described as red, or green, or blue in this projection. Every one of them should express the net state of the whole system. Therefore, every single quantum should be described as "white". However, it is possible to pass the beam through a roof prism, decomposing the projection back into the three component colors. At the output, some photons will "collapse" to the red output, others will become green, and the rest will be become blue. How is this possible? The simple explanation is that every quantum is white before the prism, but it has a partial probability of collapsing to either one of the three output bins. Suppose, for example, that a quantum detector is placed in the path of the red projection, while the other two output projections are discarded. Can we expect $100 \%$ of the input quanta to land on the "red" detector? For that to happen, the input beam would have to be $100 \%$ red, which is impossible by design. Therefore, a sharp component measurement is necessarily partial. Furthermore, if we moved 


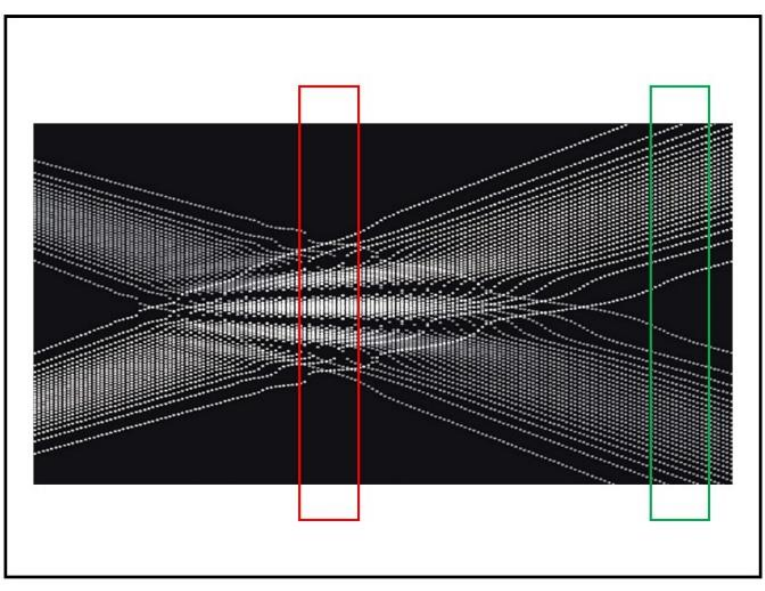

(a)

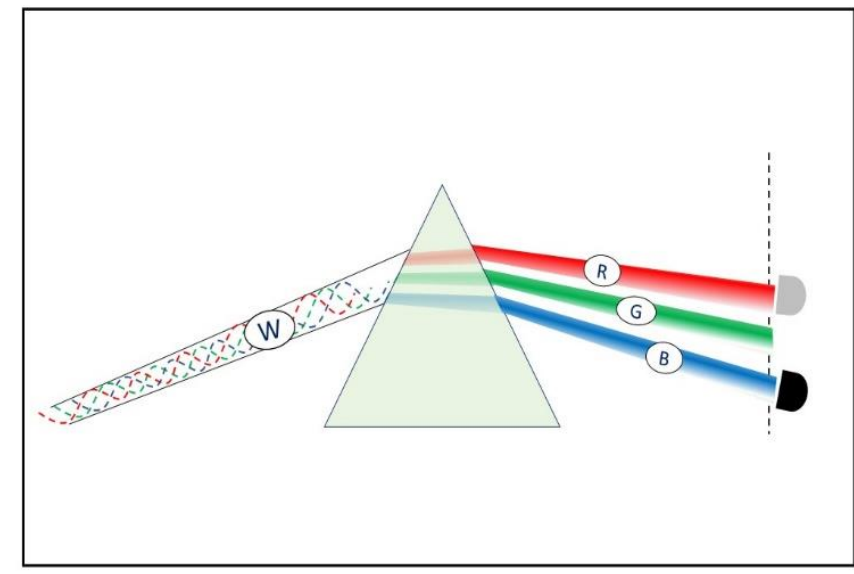

(b)

Figure 1. Statistical sub-ensembles in quantum mechanics. (a) In the case of coherent twobeam superposition, the interference volume (red box) has no physical sub-ensembles. Interference fringes can be recorded by detecting single quanta, one at a time. Yet, this quantum state has two virtual components that can be resolved in a sharp complementary measurement (green box). The absence of physical sub-ensembles does not preclude the existence of corresponding statistical sub-ensembles. (b) A white beam of light can be described as a superposition of three colors. Prior to entering the prism, every single quantum must be described as "White" and presumed to express the net effect of all the colors at the same time. At the output, a quantum can only collapse into one statistical sub-ensemble. It is impossible for all the quanta to collapse into a single color, and it is impossible for one and the same group of quanta to collapse in all the colors. Thus, different virtual components of the input state are associated with different statistical sub-ensembles at the output.

the detector from the red output to the blue output, can we expect the same exact quanta to be detected? The answer is again negative. The total sum of the output distribution must be equal to 1 . If it was the same $1 / 3$ of quanta that landed in each alternative measurement, then $2 / 3$ of quanta would magically vanish. To sum up, it is impossible for all the quanta to end up in just one output channel, and it is impossible for one and the same subgroup to be detectable in every output channel. Ergo, different partial measurements necessarily observe different sub-ensembles from the total output projection. On the other hand, if the mixed input beam was passed through a frequency convertor, it would be able to transform the full beam into a monochromatic projection. Yet, this would be a radical transformation, erasing all the information about the input beam. Ergo, transformations (that are complete) should not be confused with sharp measurements (that may be partial).

This is a very instructive conclusion. On the one hand, complex quantum states do not have physical sub-ensembles. The only correct way to predict quantum behavior is by assuming that every particle is in the net state of the prepared system (as prescribed by the corresponding wave-function). On the other hand, a quantum net state must have a set of virtual component vectors. In the event of a sharp quantum measurement, each of those vectors corresponds to a different statistical sub-ensemble. Sharp measurements provide information about "collapsed" individual components. Therefore, different sharp outputs have different statistical properties, and should be treated as different sub-populations. In plain language, when a quantum is in the net state of a projection, it must express all the components at the same time. Yet, when the measurement is designed to probe individual components, it can only have a fractional probability of producing such information, via random collapse. The only reason to expect $100 \%$ detection is in the event of a transformative "conversion" of the input state into a chosen state. Yet, such a transformation - if real - would destroy the necessary entanglement for a Bell measurement. To sum up, it is sound to maintain that "quantum theory has no physical sub-ensembles" 
in discussions about complex profiles. However, it is unsound to extend this principle to complementary "collapsed" configurations with observable statistical sub-ensembles.

\section{Is fair sampling possible in Bell experiments?}

The popular opinion in the physics community is that Bell measurements always target one and the same population in full (or, at least, sample it without bias). In practice, large $N$ quantum behavior is almost always analyzed in terms of single entities (as in "let us consider two entangled particles"). Given that every quantum is governed by the full wave-function at the input, this may seem appropriate. However, as shown in the previous section, sharp measurements involve partial observations, entailing conditional probabilities of detection. If every input particle is treated as detectable, then normalized output probabilities end up interpreted as if they apply to one and the same population by default. The outcome is a set of alternative predictions that cannot be reconciled in a physically meaningful way (either with each other, or with the formalism that predicted them). For example, Santos made a very interesting analysis in 1991 [31], explicitly suggesting that Bell violations are non-physical in quantum theory. The quantitative and qualitative aspects of this argument are equally instructive, as summarized below.

Following the well-known analysis of Clauser and Horne [32], Santos began with the general case of two systems, measured separately for two dichotomic ("yes"|"no") observables at a time. Four different experiments are required, where $p\left(a_{1}, b_{2}\right), p\left(c_{1}, d_{2}\right), p\left(a_{1}, d_{2}\right)$, and $p\left(c_{1}, b_{2}\right)$ are joint probabilities of getting the answer "yes" in both measurements, for each iteration. A condition of this analysis is that single probabilities $p\left(a_{1}\right)$ and $p\left(b_{2}\right)$ remain constant, regardless of the choice of joint measurement. Under these constraints, the following Bell-type inequality applies to any local hidden-variable model:

$$
p\left(a_{1}\right)+p\left(b_{2}\right) \geq p\left(a_{1}, b_{2}\right)+p\left(a_{1}, d_{2}\right)+p\left(c_{1}, b_{2}\right)-p\left(c_{1}, d_{2}\right) .
$$

As it is known, quantum theory entails contradictions with this inequality, but the physical significance of these predictions is up for debate, as shown by Santos. The problem starts with the definition of quantum probabilities. For instance, if $p\left(a_{1}\right)$ is defined as the ratio of detected "yes" events to the total emitted particles in the first arm of the experiment, then quantum theory does not predict a violation. To prove this, Santos calculates the expected probabilities in the classical Aspect experiments [22-24], noting that entangled photons with zero total angular momentum are in a state of spherical symmetry. This means that the photons are not localized but spread in the form of a spherical wave. Hence, the ideal probabilities of detection (without polarizers) are constrained by the solid angle $\Omega$ covered by the apertures of the lens systems and by the quantum projection operator $U_{i}$ corresponding to the observable localization of the photon in the given solid angle. Thus, for two perfect detectors in the direction of the unit vectors $u_{1}$ and $u_{2}$, the individual probabilities of observation (one at a time and two in coincidence) become:

$$
\begin{gathered}
P\left(u_{1}\right)=\left\langle\psi\left|U_{1}\right| \psi\right\rangle=\Omega / 4 \pi \\
P\left(u_{1}, u_{2}\right)=\left\langle\psi\left|U_{1} U_{2}\right| \psi\right\rangle=(\Omega / 4 \pi)^{2} \alpha(\theta, \varphi),
\end{gathered}
$$

where $|\psi\rangle$ is the Hilbert space vector corresponding to the two-photon state, ( $\cos \theta \equiv u_{1} \cdot u_{2}$ ), $\varphi$ is the half-angle of the cone covered by the corresponding apertures, and $\alpha(\theta, \varphi)$ is a correlation function that is explained in the source text. Notably, in the event of measurement with ideal polarizers, the single and joint probabilities become:

$$
\begin{gathered}
p\left(u_{1} a_{1}\right)=\left\langle\psi\left|U_{1} A_{1}\right| \psi\right\rangle=\Omega / 8 \pi \\
p\left(u_{1} a_{1}, u_{2} b_{2}\right)=\left\langle\psi\left|U_{1} A_{1} U_{2} B_{2}\right| \psi\right\rangle=(\Omega / 8 \pi)^{2} \alpha(\theta, \varphi)[1+F(\theta, \varphi) \cos 2(a-b)],
\end{gathered}
$$

where $F(\theta, \varphi)$ describes changes in polarization correlation when the angle between the relevant wave vectors is different from $\pi$. (Incidentally, this function $F$ is defined by 
Santos only for the case of atomic cascades, but it can be extended to all types of EPR experiments with singlet states. In this case, the relevant aspect is that angular dependence is not a consequence of measurement imperfection. It is a fundamental property of electromagnetic waves that linear polarization is only defined in directions perpendicular to the corresponding wave vector).

The crucial element of this analysis is that expressions (4) and (5) correspond to "correct quantum probabilities, obtained by means of the standard quantum rules for the calculation of expectation values" [op . cit]. However, if they are plugged into expression (1), then it turns out that they cannot violate the inequality. As it is well known, Bell violations are predicted for the following expressions:

$$
\begin{gathered}
p\left(a_{1}\right)=\left\langle\psi\left|A_{1}\right| \psi\right\rangle=\frac{1}{2}, \\
p\left(a_{1}, b_{2}\right)=\left\langle\psi\left|A_{1} B_{2}\right| \psi\right\rangle=\frac{1}{4}[1+\cos 2(a-b)] .
\end{gathered}
$$

In order to obtain such a profile, it is necessary to divide expressions (4) and (5) above by expressions (2) and (3) respectively. This is unusual because the presented derivation applies to ideal experiments and ideal preparations. According to Santos, the ratio between the rate of expected events and the rate of prepared quanta is the physically meaningful definition of probability (with fair sampling). Instead, Bell violations are obtained by narrowing down the analysis to "the ensemble of photon pairs, such that both members of the pair enter the corresponding apertures". This description is contested by Santos as non-physical, on the grounds that it contradicts the essence of quantum theory. The problem is that quantum mechanics forbids any consideration of hypothetical transit coordinates of photons prior to measurement, given that such positions are not subject to measurement. Furthermore, Santos notes that "the two-photon state with zero angular momentum is a pure state according to quantum mechanics and, therefore, should not be considered as composed of several distinguishable subensembles". Thus, we have a set of predictions that neither apply to the full input beam (because they are conditional), nor can they apply to possible components, because such components appear to be ruled out by quantum theory.

At first sight, Santos' conclusion sounds counterintuitive. How can it be "non-physical" to consider the apertures of an optical system, if such devices are part of the measurement preparation? Even if the quanta cannot have classical trajectories, they must obey the net state of the wave-function. Yet, filtering devices necessarily affect the profile of the wave-function, with corresponding influence on the expected probabilities of detection. Nonetheless, this analysis must be evaluated in the context of its own scientific tradition. The relevant social fact here is that Bell violations are widely perceived as if they apply to complete input projections. For several decades, the theoretical basis for "fair sampling" in Bell experiments has gone unchallenged, which is why "component switching" was only deemed relevant as a possible loophole around the predictions of quantum theory. However, if these theoretical predictions only apply to identifiable sub-ensembles, and different experiments target different sub-ensembles, then it is indeed non-physical to describe Bell violations as properties of the whole input two-photon state. As it is known, Bell violations emerge from statistically incompatible distributions, and it is impossible to reduce them to common populations without abstract concepts (such as negative probabilities) that can aptly be described as non-physical.

Another important issue is Santos' concern about the possibility of sub-ensembles in the context of quantum theory. Indeed, the absence of physical sub-ensembles is the definitive feature of superposed quantum projections. However, quantum theory also includes complementary configurations for sharp measurements. Whether quanta "evolve to" or "collapse into" such output spectral components, the point remains that well defined sub-ensembles exist in quantum theory. The caveat is that quantum sub-ensembles cannot be interpreted as classical physical sub-ensembles. Individual quanta must express the "full state" of the wave-function at the input, yet they also have fractional probabilities of detection in any one of several spectral components after wave-function 
decomposition. Consequently, the correct interpretation is not that quantum states contain physical sub-ensembles, but rather that they contain virtual spectral components with corresponding statistical sub-ensembles at the output. In other words, detected quanta do not reveal their individual history (as one might presume in the case of classical trajectories). Instead, they produce information about corresponding spectral components of their governing wave-functions. Therefore, it is perfectly "physical" to have such predictions in quantum theory. Though, with respect to quantum non-locality, the relevant implication is that different measurements isolate different statistical sub-ensembles. Therefore, there is no basis for expecting alternative partial measurements to produce fair samples of the full input population.

\section{Spin $1 / 2$ measurements are partial in quantum theory}

As shown earlier in this text, it is incorrect to interpret Bell measurements as operations that split a full projection 50-50. This type of measurement would correspond to a destructive transformation of the input profile, erasing any information about its spectral structure. Instead, Bell correlations must be interpreted as relationships between virtual components of quantum states, corresponding to output statistical sub-ensembles. For instance, if we assume a coarse division of an input beam into 12 virtual components with equal magnitude, then every output ensemble must contain 1/12 of the input number of quanta. However, alternative measurement preparations do not commute. Therefore, the same pool of quanta must be redistributed at the output, with the implications that small sub-ensembles from each output ensemble can produce coincident detection events. This is not possible in classical models, where physical ensembles are presumed to be real. In such contexts, either alternative sharp measurement target different sub-groups with disjoint distributions, or they target the same sub-group in full expecting joint distributions. In both cases Bell violations are impossible unless some sort of "spooky action at a distance" is invoked. Consequently, established misconceptions regarding the possibility of Bell violations for identical sub-ensembles do not follow from the known features of quantum theory, but rather from their unsuspected distortion, under the influence of preconceived "classical" notions.

As a corollary, the main reason to question the reality of quantum non-locality is not that quantum theory could be false (ontologically speaking), but rather that the meaning of the quantum formalism is distorted in a fundamental way. Mathematically, the outcomes of spin $1 / 2$ measurements are predicted as projections from a single orthonormal basis in the corresponding Hilbert space (rather than the average of every possible projection from all the relevant orthonormal bases). As shown above, this means that spin measurements are partial. The established practice of interpreting them as complete is in at odds with the principles of quantum mechanics and the details of real experiments (see the next section of this text). Yet, a more direct criticism is that such a practice results in paradoxical conclusions. In other words, the formalism of quantum mechanics appears to contradict itself, even though in fact it does not. This feature came to the surface with fresh force in a recent study by Cetto, Valdés-Hernández and de a Peña regarding the physical meaning of the spin projector operator in quantum mechanics [33]. As noted by the authors, the concept of spin in quantum mechanics has always been treated as intuitively inaccessible, and therefore it is accepted as an abstract quantity that only inhabits its own Hilbert space [34]. Nonetheless, they argue that it is possible to extract revealing clues about its physical essence by examining the algebraic scaffolding behind the better-known aspects of this operator. Namely, if the entangled singlet state of two spin $1 / 2$ particles is defined with the familiar expression

$$
\left|\Psi^{0}\right\rangle=\frac{1}{\sqrt{2}}\left(\left|+{ }_{r}\right\rangle\left|-{ }_{r}\right\rangle-\left|-_{r}\right\rangle\left|+{ }_{r}\right\rangle\right),
$$

then the quantum correlation $C_{Q}$ between their spin measurement $\sigma$ in two arbitrary directions $\boldsymbol{a}$ and $\boldsymbol{b}$ are given by: 


$$
C_{Q}(\boldsymbol{a}, \boldsymbol{b})=\left\langle\Psi^{0}|(\widehat{\boldsymbol{\sigma}} \cdot \boldsymbol{a}) \otimes(\widehat{\boldsymbol{\sigma}} \cdot \boldsymbol{b})| \Psi^{0}\right\rangle .
$$

In order to expose the physical content behind this expression, Cetto et all proposed to look at this problem from a different angle. Hence, it is possible to construct a new orthonormal basis for this bipartite system:

$$
\begin{aligned}
& \left|\phi^{1}\right\rangle=\left|+_{a}\right\rangle\left|-{ }_{b}\right\rangle, \\
& \left|\phi^{2}\right\rangle=\left|--_{a}\right\rangle\left|+{ }_{b}\right\rangle, \\
& \left|\phi^{3}\right\rangle=\left|+_{a}\right\rangle\left|+{ }_{b}\right\rangle, \\
& \left.\left|\phi^{4}\right\rangle=|-|-_{a}\right\rangle\left|-{ }_{b}\right\rangle,
\end{aligned}
$$

with the eigenvalue equations

$$
\begin{array}{ll}
\widehat{\boldsymbol{\sigma}} \cdot \boldsymbol{a}\left| \pm_{a}\right\rangle=\alpha\left| \pm_{a}\right\rangle, & \alpha= \pm 1, \\
\widehat{\boldsymbol{\sigma}} \cdot \boldsymbol{b}\left| \pm_{b}\right\rangle=\beta\left| \pm_{b}\right\rangle, & \beta= \pm 1 .
\end{array}
$$

This basis can then be used to derive an alternative expression for the quantum correlation equation (9):

$$
C_{Q}(\boldsymbol{a}, \boldsymbol{b})=\sum_{k} A_{k}(\boldsymbol{a}, \boldsymbol{b}) C_{k}(\boldsymbol{a}, \boldsymbol{b})
$$

where

$$
A_{k}=\alpha_{k} \beta_{k}
$$

with $\alpha_{k}$ and $\beta_{k}$ denoting the corresponding eigenvalues of bipartite state $\left|\phi^{k}\right\rangle$, and

$$
C_{k}=\left|\left\langle\phi^{k} \mid \Psi^{0}\right\rangle\right|^{2} .
$$

The advantage of this reformulation is that the two coefficients of expression (16) have an explicit physical meaning. $A_{k}$ is the eigenvalue of the operator $(\widehat{\boldsymbol{\sigma}} \cdot \boldsymbol{a} \otimes \widehat{\boldsymbol{\sigma}} \cdot \boldsymbol{b})$ for the bipartite state $\left|\phi^{k}\right\rangle$, while $C_{k}$ is the relative weight of the eigenvalue $A_{k}$. Furthermore, $C_{k}$ are nonnegative and their sum is

$$
\sum_{k} C_{k}=\sum_{k}\left\langle\Psi^{0} \mid \phi^{k}\right\rangle\left\langle\phi^{k} \mid \Psi^{0}\right\rangle=1 .
$$

Therefore, as shown by Cetto et al. in the quoted text, $C_{k}$ is the joint probability of $A_{k}$ :

$$
C_{k}(\boldsymbol{a}, \boldsymbol{b})=P_{a b}^{k}(\alpha, \beta) .
$$

The important nuance here is that the coefficients $A_{k}$ and $C_{k}$ are explicitly anchored to the chosen basis $\left\{\left|\phi^{k}\right\rangle\right\}$ that, in turn, is constructed from the individual eigenvectors $\left| \pm_{a}\right\rangle$ and $\left| \pm_{b}\right\rangle$. Because of their dependence on $\boldsymbol{a}$ and $\boldsymbol{b}$, every new joint measurement would be associated with its own eigenvalues and relative weights. Therefore, any two non-identical joint measurements must have incommensurable coefficients. For instance, even when a single direction is part of two different joint measurements - such as $(\boldsymbol{a}, \boldsymbol{b})$ and $\left(a, b^{\prime}\right)$ - it will be associated with two different types of coefficients, which cannot be mixed together.

The authors further explain that $C_{Q}(\boldsymbol{a}, \boldsymbol{b})$, as defined in equation (16), corresponds to a partitioning of state vector $\left|\Psi^{0}\right\rangle$ into four mutually exclusive and complementary subensembles with the outcomes $(+,-),(-,+),(+,+)$ and $(-,-)$, but only for the specified basis vectors $\left|\phi^{k}\right\rangle, \mathrm{k}=1,2,3,4$. Accordingly, the corresponding partitioning of the probability space $\Lambda$ must be expressed as

$$
C_{Q}(\boldsymbol{a}, \boldsymbol{b})=\sum_{k} \int_{\Lambda_{k}} A_{k}(\boldsymbol{a}, \boldsymbol{b}, \lambda) \rho(\lambda) d \lambda,
$$

with

$$
\Lambda_{k}=\Lambda_{k}\left(\boldsymbol{a}, \boldsymbol{b}, \alpha_{k} \beta_{k}\right)
$$


and

$$
C_{k}(\boldsymbol{a}, \boldsymbol{b})=\int_{\Lambda_{k}} \rho(\lambda) d \lambda .
$$

The significance of this exercise becomes evident when expression (21) is plugged into the CHSH equation [21] (since it is the one most frequently used in Bell-type experiments):

$$
\begin{aligned}
C_{Q}(\boldsymbol{a}, \boldsymbol{b}) & +C_{Q}\left(\boldsymbol{a}, \boldsymbol{b}^{\prime}\right)+C_{Q}\left(\boldsymbol{a}^{\prime}, \boldsymbol{b}\right)-C_{Q}\left(\boldsymbol{a}^{\prime}, \boldsymbol{b}^{\prime}\right) \\
& =\sum_{k} \int_{\Lambda_{k}} \alpha_{k} \beta_{k} \rho d \lambda+\sum_{l} \int_{\Lambda_{l}} \alpha_{l} \beta^{\prime}{ }_{l} \rho d \lambda+\sum_{m} \int_{\Lambda_{m}} \alpha_{m}^{\prime} \beta_{m} \rho d \lambda-\sum_{n} \int_{\Lambda_{n}} \alpha_{n}^{\prime}{ }_{n}{ }^{\prime}{ }_{n} \rho d \lambda .
\end{aligned}
$$

As explained above, each joint measurements partitions the probability space $\Lambda$ according to its own parameters. Therefore, it would be incorrect to group these four terms under a single integral sign, as it is done in the analysis of Bell correlations $C_{B}$ :

$$
\begin{aligned}
C_{B}(\boldsymbol{a}, \boldsymbol{b})+C_{B}\left(\boldsymbol{a}, \boldsymbol{b}^{\prime}\right)+C_{B}\left(\boldsymbol{a}^{\prime}, \boldsymbol{b}\right)-C_{B}\left(\boldsymbol{a}^{\prime}, \boldsymbol{b}^{\prime}\right) \\
=\int_{\Lambda}\left[\alpha \beta+\alpha \beta^{\prime}+\alpha^{\prime} \beta-\alpha^{\prime} \beta^{\prime}\right] \rho(\lambda) d \lambda \\
\quad=\int_{\Lambda}\left[\alpha\left(\beta+\beta^{\prime}\right)+\alpha^{\prime}\left(\beta-\beta^{\prime}\right)\right] \rho(\lambda) d \lambda \leq 2 .
\end{aligned}
$$

As a corollary, quantum correlations are naturally able to violate Bell-type inequalities due to the statistical incompatibility between the corresponding probability spaces. There is no need to stipulate some sort of mysterious quality (such as non-locality) for the explanation of quantum behavior. However, if we try to identify the source of this incompatibility, then we get a paradox. According to Cetto and collaborators, $\left\{\Lambda_{k}\right\},\left\{\Lambda_{l}\right\},\left\{\Lambda_{m}\right\}$, and $\left\{\Lambda_{n}\right\}$, represent exhaustive subdivisions of the probability space $\Lambda$ into relevant sectors. In other words, one and the same probability space is broken down in alternative ways without "losses". If that was the case, then we would get physical incompatibility between measurements, but not statistical incompatibility. After all, independent exhaustive measurements result in joint distributions, and it is a mathematical fact that joint distributions cannot violate Bell-type inequalities [35]. So, how is it possible that a correct mathematical demonstration produces an outcome that is mathematically impossible?

When there is a paradox, the most likely reason is that some important piece of information is either missing or distorted. In this case, the paradox vanishes if spin measurements are assumed to be partial, rather than complete. Therefore, the culprit is the established tradition to deny the possibility of sub-ensembles in quantum mechanics. A single quantum must be described as if it is in the full state of the wave-function. However, sharp measurements unavoidably entail fractional probabilities of collapse. As seen in the preceding analysis, the singlet state is spherically symmetric, but spin measurements are well-defined only for individual wave-vectors. Indeed, a single quantum is in the full state of the wave-function at the source, but it can only collapse to a single point from a wide projection on a screen. Accordingly, there is a physical difference between "measuring a quantum state in four alternative ways" and "measuring a single quantum in four alternative ways". The subtle but important difference is that conditional measurements have partial probability of manifestation. Therefore, it cannot be the case that one and the same quantum particle is always detectable in any spin measurement.

To sum up, Cetto et al. arrived at a paradoxical conclusion because they respected the established tradition and assumed that the same space $\Lambda$ is exhaustively partitioned in incompatible ways by different joint measurements. Yet, just because an eigenbasis can form a complete and orthonormal set, it does not follow with necessity that it can be used to describe the complete profile of the input quantum state $\left|\Psi^{0}\right\rangle$. Absent any proof that such an assumption is correct, a more plausible explanation is that every eigenbasis defines a different sub-space that, in turn, is partitioned as described. In other words, different joint measurements produce different partitions that - together - define non-identical probability sub-spaces from a larger global probability space. The precursor to such an outcome is that every single measurement must delineate a slice of the global probability space associated with it, and the overlap between any two such slices is what allows for a 
joint measurement to be predicted. For instance, the slice associated with a sharp measurement in direction $a$ must have different volumes of overlap with corresponding slices for $\boldsymbol{b}$ and $\boldsymbol{b}^{\prime}$. Accordingly, different joint measurements correspond to different volumes of overlap. It is the spatial inconsistency between these post-selected volumes that explains their statistical incompatibility. The bottom line is that a projection in a single orthonormal basis must correspond to a component measurement from a complex input quantum state. In contrast, a full measurement (or, rather, a full transformation of the input state) would have to correspond to the weighted average of all the projections from every valid orthonormal basis into a single direction. As it is now, conditional quantum measurements are described as complete, with paradoxical implications. In the same vein, loophole-free Bell experiments are designed to "confirm" the fair-sampling assumption, only to discover that such a demonstration would require signaling non-locality.

\section{Spin $1 / 2$ measurements are partial in quantum experiments}

Another popular opinion in the physics community is that non-commuting variables always apply to one and the same population. Accordingly, spin measurements should always target the same population because they probe non-commuting variables. At first sight, this belief has merit, especially if one considers canonical conjugates, such as position and momentum. All the momentum vectors are superposed with each other, and all the position vectors are superposed with each other, as part of a wave-function profile, but they are nonetheless alternative spectra. Each spectrum exhausts the physically possible values of a complete system for each variable. Passing from one distribution to the other requires a physical transformation (mathematically expressed by a Fourier transform). However, spin $1 / 2$ vectors are superposed directly with each other, as part of a wavefunction profile (much like the colors of a rainbow). Therefore, they form a quantum system together, and none of them can exhaust a full profile individually. The curious feature is that spin $1 / 2$ variables also do not commute, and therefore cannot be separable from each other. Still, their non-commutativity follows from their limited distinguishability, proportional to the angle between any two vectors. Thus, spin $1 / 2$ projections have variable degrees of commutativity [19]. The implication is that binary spin $1 / 2$ measurements target two orthogonal sub-ensembles (the only ones that are distinguishable), rather than split the full population 50-50. Unfortunately, this feature is overlooked in numerous theoretical studies on this topic, as shown in the preceding two sections of this text.

Typical examples of spin $1 / 2$ variables include electron spin and photon polarization. For example, a depolarized optical beam contains every possible state of linear polarization in superposition. This means that every single quantum is simultaneously expressing the net effect of all those vectors. Accordingly, every single quantum has a fractional probability of collapsing to any component. Yet, this property is often overlooked, because of the perception that linear polarization is measured directly with polarizing beam-splitters (PBS), as it is done in classical mechanics. In other words, $100 \%$ of quanta are presumed to be split 50-50 at any possible PBS angle of alignment. Consequently, alternative measurements are presumed to sample one and the same complete projection. On closer inspection, this belief is not supported at all by the details of actual quantum experiments. 


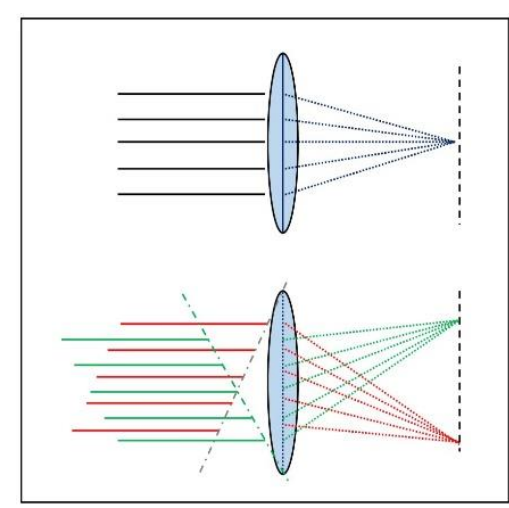

(a)

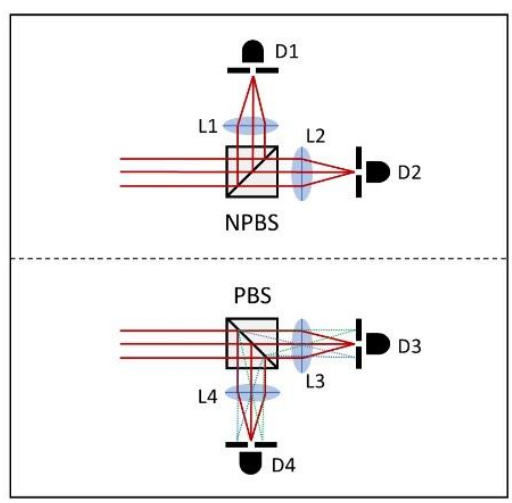

(b)

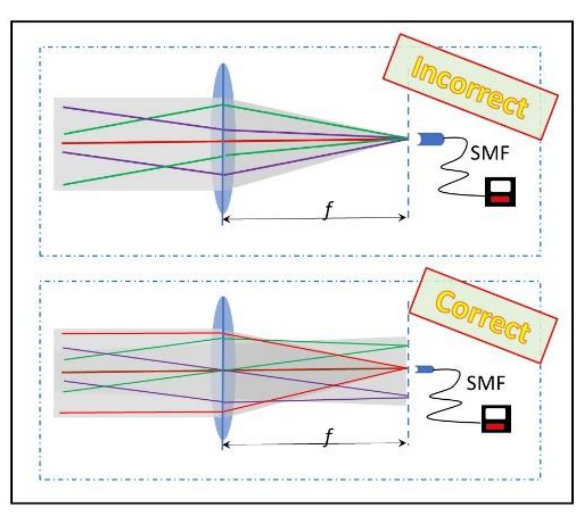

(c)

Figure 2. Component selection in optical Bell experiments. (a) A collimated quantum beam (top image) can be focused onto a single point in the focal plane of a lens, just like a classical plane wave. If arrays of phase delays are introduced for various sub-components (bottom image), the beam can no longer be focused onto a single point. (b) Top image: a single-mode depolarized beam is split by a non-polarizing beam-splitter (NPBS). Each output projection is focused onto a single corresponding point. Bottom image: in contrast, a similar beam must suffer a different fate if split by a polarizing beam-splitter (PBS). Because of birefringence, different polarization components of the governing wave-function are delayed relative to each other. This effect adds new components to the momentum spectrum. Each output projection is linearly polarized, but each momentum component carries information about a corresponding polarization vector from the input projection. Relevant statistical sub-ensembles can be isolated with pinholes. (c) Modern experiments use optical fiber tips, instead of pinholes, to isolate relevant spatial modes. Top image: it is a common misconception that multi-mode beams can be focused onto a single point, to be collected by a single mode optical fiber (SMF). Bottom image: instead, the combination of lens and SMF works as a spatial filter. Each momentum component projects to a different point in the focal plane. The SMF isolates only one such component for measurement.

To keep things simple, let us consider an optical projection that is produced by mixing only three coherent components. These components are linearly polarized in three different planes: vertical, horizontal, and diagonal. Given their state of superposition, the three components are now indistinguishable. According to the principle of quantum superposition, $100 \%$ of the quanta are in the net state of the projection. None of them is just diagonal, or vertical, or horizontal. Still, it should be possible to make a sharp measurement that decomposes the net state back into its virtual components. Therefore, it should be possible to design a measurement set up in which individual components are sampled in pure form, but how? If the beam is passed through a PBS, it will be split in only two projections, but there are three components. For example, if the PBS is oriented with the fast axis in the vertical plane, then the vertical component will be transmitted; the horizontal component will be reflected, but the diagonal component will be split 50-50. Therefore, either output will sample two virtual components of the net state. If the PBS is rotated to any other angle, the output mixture becomes different in each channel, but no setting is possible in which only one component of the input spectrum is isolated in pure form. In short, polarizing beam-splitters cannot isolate individual component vectors. They can only erase the input state, transforming it into a double projection.

How do quantum measurements isolate individual polarization components? The answer is that they combine the properties of beam splitters with the properties of spatial filters $[36,37]$. If a single mode (collimated) optical projection passes through a birefringent medium, then different polarization components are likely to experience different velocities of propagation. For instance, a vertical component might take the fast axis, while the horizontal component takes the slow axis. In a PBS, these two components are directed 
into different channels, but what happens to the intermediate states of polarization? The most obvious explanation is that the diagonal component is going to be split 50-50, but it will have to experience a rotation before it is either transmitted or reflected. Hence, the diagonal component may be transmitted with a delay, relative to the fast channel, or it can be reflected faster than the slow component. In both cases, there is a polarizationdependent phase delay at the output (Fig. 2). If the whole projection was in phase, it would be focused on a single focal point. When some components are out of phase, the output projection is no longer in a single optical mode of propagation. It must generate several focal points, if passed through a lens. Therefore, a pinhole can be used to isolate a single mode of propagation, which can provide information about a single virtual component from the input polarization profile. This is why successful Bell experiments with photons always employ PBSs and lenses, before isolating a single point for detection from a much wide projection. In the past, these points were isolated with pinholes. Modern experiments use single-mode optical fibers that can isolate such components with higher degrees of purity (Fig. 2c). In short, quantum spin measurements are not intended to erase input profiles and to transform them into 50-50 projections. They are intended to isolate two orthogonal components from a long list of component vectors. Consequently, different measurements target different sub-ensembles from a wider output projection.

To sum up, there is a major gap between quantum behavior as commonly described and quantum behavior as it can be inferred from known theoretical and experimental facts. Bell correlations are predicted and confirmed for virtual wave-function components but are described as if they apply to complete wave-function transformations. The principle that "there are no sub-ensembles in quantum theory" appears to prevent the acknowledgement of statistical sub-ensembles, even in the face of strong theoretical and experimental evidence to the contrary. An important implication of this conclusion is that loophole-free Bell experiments are misguided, insofar as they try to prove the reality of nonlocality and the validity of quantum theory at the same time. The two concepts are fundamentally incompatible, as seen in the paradoxical outcome that Bell violations and signaling non-locality must be real at the same for such expectations to be confirmed.

\section{Pre-selected quantum coincidences}

Signaling non-locality is impossible in quantum theory. Accordingly, coincident subensembles must be smaller than the ensembles of single events, as argued above. Yet, this leads to the question: how do loophole-free experiments confirm quantum predictions without post-selection? In particular, how is it possible violate the CHSH inequality when $100 \%$ of the detected events are coincident? The answer is that such experiments are conducted by extracting photons from correlated atomic systems. As will be shown below, this method enables the pre-selection of coincident events via "temporal compression" without ever recording unpaired events. In a nutshell, these experiments cheat by using heralded detection schemes to preselect coincidences in a biased way. Single events are excluded from detection, but this does not reveal anything new about the ontological features of our Universe.

For an intuitive grasp of this type of contexts, consider an optical projection detected on a screen. The observable pattern can be expressed as a distribution, with a probability value for each coordinate. Every possible value corresponds to a fraction of expected detection events in a fixed unit of time. The number of registered photons at each point is proportional to the magnitude of the corresponding component vector in the input projection. Coincident events can be downselected from the data generated with two such screens, by choosing relevant points of detection (one on each screen). Each coordinate receives a large set of single events, enabling the post-selection of a relevant coincident subset. Let us suppose, for the purpose of the argument, that it is possible to violate the CHSH inequality without post-selection in such a context, with unconditional detection. Every single event at a point on detector A has a matching partner at a point on detector B. This would satisfy the Larsson criterion, suggesting that different measurements 
sample the same population. It is important to point out that this criterion is very powerful in this context, removing any concern about "hidden variables". For example, suppose that quantum measurements are perturbative. Changing the polarization settings modulates the input beam, and we can take it for granted that a different wave-function component will hit the chosen point of detection for each measurement. This would be irrelevant if all the photons (or at least $82.8 \%$ of them) have matching partners at both points of detection [26]. After all, Bell experiments require Alice to keep a fixed detection set-up, while Bob switches between two possible variables (and vice versa). The only way to obtain a violation is if resulting correlations require changes in the distribution of Alice photons. This would require the photons registered by Alice to "know" what happens to the photons registered by Bob. No plausible mechanism for "component switching" is available in this context. Accordingly, violations of the CHSH inequality without post-selection in such optical projections would necessarily confirm the possibility of signaling non-locality and falsify quantum theory.

Though, an important aspect of this description is that it presumes unrestricted sampling of spatial distributions. Quantum systems also allow for experimental settings in which temporal distributions are observable. To clarify the difference, consider a single photon propagating as part of a complex optical projection. In a sharp spatial measurement, the quantum must collapse to one possible value in the output distribution. The probability of it landing at any point is fractional, but the total probability of it landing somewhere on the screen is unitary. In contrast, consider an atom with a single electron on the outer shell. A polarized laser can be used to pump it into a higher energy state, causing the emission of a photon with predetermined linear polarization. However, the spin state of the electron is undefined. Ergo, it is not always available for excitation in the chosen plane. Essentially, the electron must first "collapse" into the chosen spin state (at an unpredictable moment in time). Only then it can become excited enough to eject a photon. Thus, at any chosen time coordinate, within the interval of excitation, there is a fractional probability of $\gamma$-emission, while the total probability in a predictable time window is $100 \%$. This analogy can be extended to joint measurements. Hence, if Alice and Bob were to make instantaneous measurements from two correlated atoms, they would get many null events, a large number of single events, and a small number of coincident events, just like in the experiments with spatial distributions. The crucial difference is that Alice and Bob can increase the time-window of detection, effectively detecting all the pairs. This would be like using a bucked detector, instead of a pinhole detector, in a spatial experiment. All the pairs are detected in coincidence. Ergo, it seems that Bell violations by local means should be impossible. Notably, they are also impossible in quantum theory. Incompatible sharp measurements correspond to incompatible component vectors from the input state. Measuring all the events lumps all the components together. Thus, it appears that only signaling non-locality can produce incompatible distributions for pairwise measurements in this arrangement. Yet, there is a way to avoid this constraint.

The curious thing about temporal distributions is that they allow for measurement schemes in which the window of coincidence is remotely manipulated, by taking advantage of quantum entanglement. Hence, a single atom has an unpredictable moment of photonic emission in the chosen plane of excitation. However, if two atoms are entangled, then they acquire the ability to "collapse" in synch. Individual moments of collapse are still associated with fractional probabilities, but the conditional probability can be certain. In other words, if Alice and Bob choose to do the same exact measurement, they should expect simultaneous detection events. Accordingly, it is possible to prepare a detection scheme in which detection events in Allice's apparatus trigger a heralded detection in Bob's apparatus (Fig. 3). For clarity, an event in Alice's terminal triggers a "Start recording" instruction at Bob's terminal, followed by a "Stop recording" instruction as soon as a detection event has occurred. This can be described as "temporal pinhole", given that the window of detection can be reduced in the same way in which a spatial region of detection is reduced by an actual pinhole. Furthermore, if Alice and Bob make non-identical measurements, their required average window of detection will gradually increase 


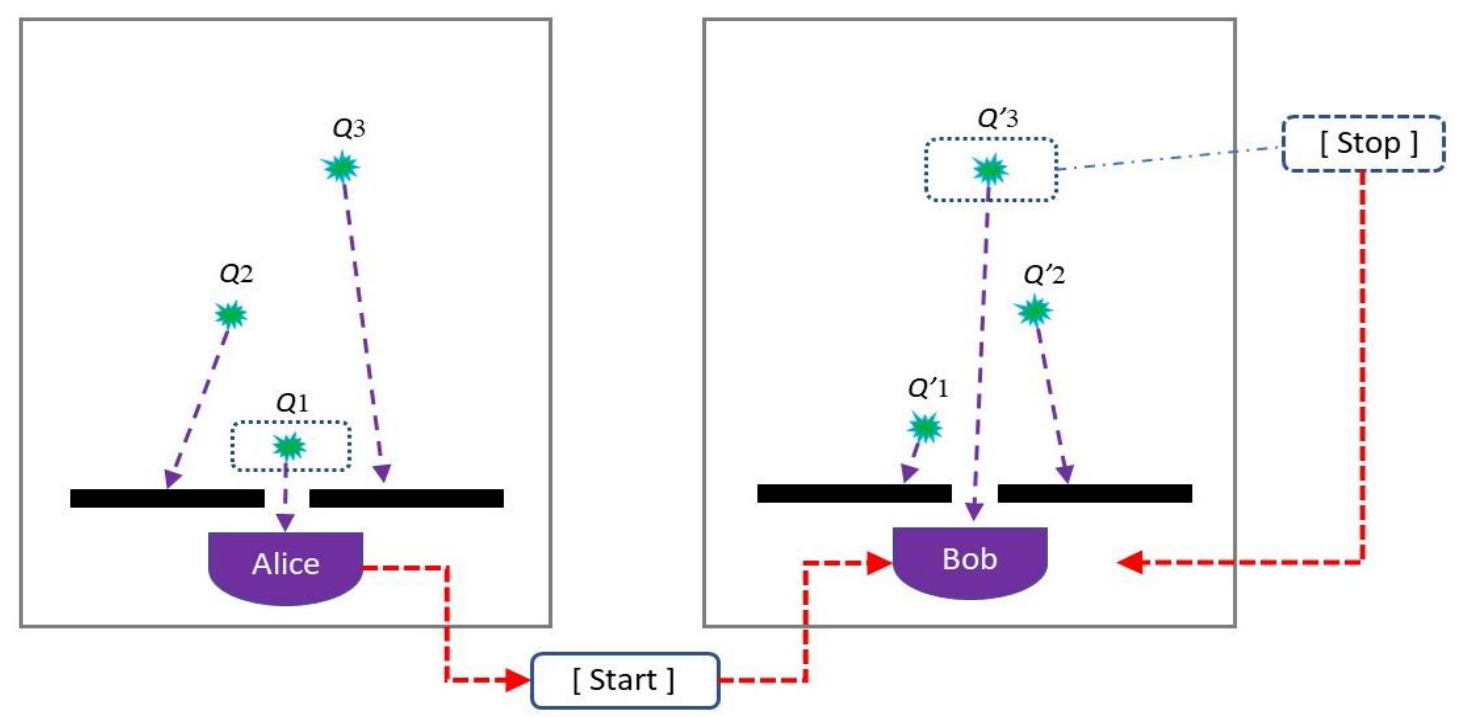

Figure 3. Heralded detection dilutes quantum entanglement. When Alice detects a quantum, Bob is very likely to have an entangled partner in his set-up. However, Bob's quantum is not necessarily detectable. This explains the large proportion of single events in typical Bell experiments. (The effect of background noise and detector inefficiency is relatively small). Heralded detection aims to mimic the conditions for "fair sampling" by eliminating single events. Every time Alice detects a quantum, Bob receives a "start recording" instruction, followed by a "stop recording" instruction upon detection. If Alice's quantum partner is detectable, then a true entangled pair is registered. Otherwise, Bob must wait until any other quantum is detected, as shown. This results in a hybrid sample, with a small number of entangled pairs and a large number of spurious coincidences. It is still possible to achieve statistically significant Bell violations, but the magnitude is dramatically reduced by the addition of non-entangled pairs. Heralded detection can only achieve perfect "fair sampling" if entangled pairs are eliminated entirely, in which case Bell violations become impossible. This is why "loophole-free" experiments do not falsify quantum theory.

(up to a limit) in direct proportionality with the difference in their chosen angles of measurement. If Alice makes a measurement $\boldsymbol{a}$, then Bob can make an adjacent measurement $\boldsymbol{b}$ in a very narrow coincidence window, with high probability. Getting a coincident event in this scheme is a compression of numerous possible combinations, including null events and single events. However, this is a partial compression, because the "start" - "stop" recording instructions for Bob begin when Alice has a detection event and have a very high probability of stopping shortly after that. Consequently, every joint event is a partial and biased scan of the corresponding probability space. Even if Bob keeps a fixed measurement setting, his events will belong to different "temporal slits", depending on Alice's choice. For example, imagine that Alice has a choice between pumping her atom at $+45^{\circ}$ or $-45^{\circ}$, while $\mathrm{Bob}^{\prime}$ s laser is fixed at $0^{\circ}$. Bob's detector will be heralded by different types of events in each case. If Alice chooses $+45^{\circ}$, Bob's detector will start scanning the temporal space from a matching time coordinate. Given the entangled nature of the pumped atoms, Bob can expect an event with high probability in a very short segment of time, as soon as Alice records an event. If Alice chooses to pump her atom at $-45^{\circ}$, then Bob's detector will start scanning the corresponding temporal space from the opposite direction. Hence, Bob keeps fixed measurement settings, but does not make neutral measurements. The best way to visualize this is by assuming that Bob's atom is constantly pumped and constantly emitting or not emitting photons, but only heralded compressed events from partial temporal windows are selected for analysis. Thus, Alice and Bob can pre-select coincident ensembles of detections with the same statistical properties as post-selected events from spatial distributions. Heralded detection can also be used in regular Bell experiments with photons, with the same effect (Fig. 3). The only problem is that such a scheme is not as 
pure as the detection protocols with post-selection, because it dilutes the correlations. Thus, it cannot produce maximal violations of the CHSH inequality, but it allows for sufficient bias to ensure "statistically significant" violations. In short, this method produces variable coincidence windows, stretching the interval of observation as long as necessary to filter out single events (i.e., just enough to produce the false appearance of fair sampling in "loophole-free" Bell experiments).

To sum up, atomic systems can be subjected to sharp stimulation, in which case they have fractional probabilities of instantaneous $\gamma$-emission. This entails very small probabilities for temporally coincident detection of photons from correlated systems, especially in the case of non-identical measurements. Yet, it is possible to force the exclusive detection of double events by extending the temporal segment of detection. In the case of heralded detection schemes for non-commuting observables, this allows for biased pre-selection. By switching her measurement setting, Alice can manipulate the temporal segment in which Bob is scanning for coincident events. Thus, it is not the quanta that "know" what Alice chooses to observe. It is Bob who knows when to scan for events that are temporally adjacent to Alice's choice, given the correlation between their atomic systems. Accordingly, quantum experiments can satisfy Larsson's criterion for fair sampling without actually performing fair sampling. This is a loophole around the requirements for physical non-locality, but not a loophole around quantum theory. On the contrary, this is a loophole that is needed by quantum theory to satisfy an otherwise impossible expectation. As shown above, Bell experiments with true fair sampling can only produce violations if signaling non-locality is real. As a corollary, "loophole-free" Bell experiments that obtain Bell violations without post-selection do not demonstrate signaling non-locality and do not produce any evidence that can falsify quantum theory. They are simply designed to satisfy the appearance of non-locality, on the basis of mistaken interpretive assumptions about the reality of statistical sub-ensembles in quantum systems.

\section{Discussion}

The concept of non-locality has grown into a major source of confusion about the nature of quantum behavior. While it was motivated by real problems in quantum theory, it evolved in the context of meta-theoretical discussions that left quantum theory behind. Thus, it might be helpful to disambiguate the meanings of this concept that relate and do not relate to known facts in quantum mechanics. On the one hand, entangled quanta display patterns of behavior that can only be described as non-local. This can be seen in experiments with non-local interference $[38,39]$, where one beam passes through an interferometer and another one reveals the interference pattern. Likewise, "ghost-imaging" is a process in which one beam interacts with an object, and another beam reveals the image $[27,28]$. This behavior is non-local because predicted patterns emerge without any local interaction between the detected quanta and the corresponding objects. On the other hand, it is incorrect to describe this sort of non-locality as a non-classical feature, because similar patterns are possible in classical mechanics as well. For example, a rigid mechanism with two machine guns can be used to spray two targets with bullets. If one stream of bullets interacts with an object, a shadow of the same object can be found in the other stream, via post-selection $[15,16]$. What makes quantum behavior different from classical bullets is not "non-locality". The obvious difference is that classical behavior results from particle correlations, while quantum behavior does not. In other words, classical patterns can be explained in terms of bullets that have rectilinear trajectories. This is why they produce shadows. In contrast, single quanta reflect global interference patterns that require wave-like interactions for their explanation. The central mystery of quantum mechanics (if not the only mystery, as argued by Feynman [40]) is that single quanta somehow reproduce complex wave-function profiles, with numerous component vectors in superposition, while propagating one at a time. In the case of EPR systems, non-locality results from point-by-point correlations between the cross-sections of such evolving interference patterns. Unfortunately, the physics community was seduced by the non-local 
aspect for several generations, and this "central mystery" is still insufficiently explored. To be clear, Bell experiments inspired numerous technological and theoretical breakthroughs. The problem is not that such a project enjoyed attention. The problem is that it evolved by grossly distorting the essence of quantum theory and all the relevant experiments. Thus, its success had a detrimental effect on the study of other topics with high scientific value.

For practical purposes, the main consequence of this "central mystery" is the measurement problem. Every single quantum must be described as if it is in the net state of the whole system. However, it can only "collapse" to one component value in the event of a sharp measurement. The probability associated with any one component vector is fractional. Therefore, not every quantum is detectable after collapse in the case of a sharp measurement. On the one hand, there are no physical sub-ensembles in complex quantum states. On the other hand, every virtual component of such a state is associated with a statistical sub-ensemble in a complementary preparation. In the case of spin $1 / 2$ variables, this means that non-identical sharp measurements target non-identical statistical sub-ensembles. Ergo, sharp measurements of electron spin or photon polarization are necessarily partial. Somehow, this nuance was "lost in the math" and hasn't been noticed until loophole-free experiments produced the appearance of impossible signaling non-locality, as explained above. Theoretically, sharp component measurements cannot be complete in quantum mechanics. Yet, the predictions for Bell measurements are too often described as complete, fueling speculations about superluminal interactions. Experimentally, it is known that accurate data requires careful isolation of various sub-ensembles (either in space, or time) in relevant tests. Yet, these observations are again presented as complete. In short, there is a strong agreement between theory and experiment, but there is a strong disagreement between fact and opinion in the interpretation of quantum entanglement. This debate has been going on for generations, while both sides ( $p r o$ and contra non-locality) share the same misconceptions. Granted, it would be helpful to explore the partial nature of Bell measurements with new theoretical studies and new experiments (and hopefully this essay will encourage more of them). However, the real obstacle is interpretive. For a meaningful breakthrough in this area, it is simply necessary to call a spade a spade. Partial measurements are not complete measurements.

Funding: "This research received no external funding".

Data Availability Statement: Not applicable.

Acknowledgments: The author is grateful to all the participants of the 2021 Conference on Quantum Foundations at Linnaeus University in Växjö, Sweden. Special credit is due to Jan-Åke Larsson and Philippe Grangier for their help in understanding the subtler aspects of modern loophole-free Bell experiments.

Conflicts of Interest: "The authors declare no conflict of interest." 


\section{References}

1. Bell, J. S. Speakable and unspeakable in quantum mechanics, Cambridge, 1987.

2. Bell, M.; Gao, S. (eds): Quantum Nonlocality and Reality: 50 Years of Bell's Theorem, Cambridge, 2016.

3. Wheeler, J. A.; Zurek, W. H. (eds): Quantum Theory and Measurement, Princeton, 1983.

4. Afriat, A.; Selleri, F. The Einstein, Podolsky, and Rosen Paradox in Atomic, Nuclear, and Particle Physics, Springer, 1998.

5. Hensen, B.; et al. Loophole-free Bell inequality violation using electron spins separated by 1.3 kilometres, Nature, 2015, 526, p. 682.

6. Rosenfeld, W.; et al. Event-Ready Bell Test Using Entangled Atoms Simultaneously Closing Detection and Locality Loopholes, Phys. Rev. Lett. 2017, 119, 010402.

7. Ghirardi, G. C.; Rimini, A.; Weber, T. A. General argument against superluminal transmission through the quantum mechanical measurement process. Lett. Nuovo Cimento. 1980, 27, p. 293.

8. Simon, C.; Buzek, V.; Gisin, N. The no-signaling condition and quantum dynamics, Phys. Rev. Lett. 2001, 87, 170405.

9. Gisin, N.; et al. Constraints on nonlocality in networks from no-signaling and independence, Nat Commun, 2020, 11, p. 2378.

10. Christensen, B. G.; et al. Detection-Loophole-Free Test of Quantum Nonlocality, and Applications, Phys. Rev. Lett. 2013, 111, 130406.

11. Giustina, M.; et al. Bell violation using entangled photons without the fair-sampling assumption, Nature 2013, 497, p. 227.

12. Giustina, M.; et al. Significant-loophole-free test of Bell's theorem with entangled photons, Phys. Rev. Lett. 2015, 115, 250401.

13. Shalm, L. K.; et al. A strong loophole-free test of local realism, Phys. Rev. Lett. 2015, 115, 250402.

14. Zeilinger, A. Experiment and the foundations of quantum physics, Rev. Mod. Phys. 1999, 71, p.S288.

15. Shih, Y. The physics of 2 is not 1+1. The Western Ontario Series in Philosophy of Science 2009, 73, p. 157.

16. Shih, Y. Two-photon entanglement and quantum reality, Advances in Atomic, Molecular, and Optical Physics 1999, $41,1$.

17. Bertlman, R. A.; Zeilinger, A. (eds): Quantum [Un]speakables: From Bell to Quantum Information, Springer, 2002.

18. Peres, A. Quantum Theory: Concepts and Methods, Kluwer, 1993.

19. Seevinck, M.; Uffink, J. Local commutativity versus Bell inequality violation for entangled states and versus non-violation for separable states. Phys. Rev. A 2007, 76, 042105.

20. Wheeler, J. A.; Zurek, W. H. (eds): Quantum Theory and Measurement, Princeton, 1983.

21. Clauser, J. F.; Horne, M. A.; Shimony, A.; Holt, R. A. Proposed Experiment to Test Local Hidden-Variable Theories, Phys. Rev. Lett. 1969, 23, p.880.

22. Aspect, A.; Grangier, P.; Roger, G. Experimental tests of realistic local theories via Bell's theorem, Phys. Rev. Lett. 1981, 47, p.460.

23. Aspect, A.; Grangier, P.; Roger, G. Experimental realization of Einstein-Podolsky-Rosen-Bohm gedankenexperiment: a new violation of Bell's inequalities, Phys. Rev. Lett. 1982, 49, p.91.

24. Aspect, A.; Dalibard, J.; Roger, G. Experimental test of Bell's inequalities using time-varying analyzers, Phys. Rev. Lett. 1982, 49, p.1804.

25. Aspect, A. Three experimental tests of Bell inequalities by the measurement of polarization correlations between photons, Ph. D thesis defended in Orsay (France) on feb 1st, 1983. Also, personal communication with Philippe Grangier.

26. Larsson, J. Bell's inequality and detector inefficiency, Phys. Rev. A 1998, 57, p.3304.

27. Padgett, M. J.; Boyd, R. W. An introduction to ghost imaging: quantum and classical, Phil. Trans. R. Soc. A 2017, $375,20160233$.

28. Shih, Y. Quantum Imaging, IEEE Journal of Selected Topics in Quantum Electronics 2007, 13, p.1016.

29. Ma, X.; Kofler, J.; Zeilinger, A. Delayed-choice gedanken experiments and their realizations, Rev. Mod. Phys. $2016,88,015005$.

30. Mardari, G. How to Erase Quantum Monogamy? Quantum Rep. 2021, 3, p.53.

31. Santos, E. Does quantum mechanics violate the Bell inequalities? Phys. Rev. Lett. 1991, 66, p.1388.

32. Clauser, J. F.; Horne, M. A. Experimental consequences of objective local theories, Phys. Rev. D 1974, 10, p.526.

33. Cetto, A. M.; Valdés-Hernández, A.; de la Peña, L. On the spin projection operator and the probabilistic meaning of the bipartite correlation function, Found. Phys. 2020, 50, p.27.

34. Tomonaga, S.-I. The Story of Spin, The University of Chicago Press, Chicago, 1997.

35. Fine, A. Joint distributions, quantum correlations, and commuting observables, J. Math. Phys. 1982, 23, p.1306.

36. Hecht, E. Optics, Addison-Wesley, 2001.

37. Freegarde, T. Introduction to the Physics of Waves, Cambridge, 2013.

38. Chiao, R. Y.; Kwiat, P. G.; Steinberg, A. M. Quantum non-locality in two-photon experiments at Berkeley, Quantum Semiclass. Opt. 1995, 7, p.259.

39. Strekalov, D. V.; Sergienko, A. V.; Klyshko, D. N.; Shih, Y. H. Observation of Two-Photon “Ghost” Interference and Diffraction, Phys. Rev. Lett. 1995, 74, p.3600.

40. Feynman, R.; Leighton, R.; Sands, M. The Feynman Lectures on Physics, California Institute of Technology, 1963, vol. 1, ch. 37. 